\title{
CLINICAL AND RADIOGRAPHICAL EVALUATION OF BIOCERAMIC ROOT CANAL SEALER AND MTA IN APEXIFICATION OF IMMATURE PERMANENT TEETH
}

\author{
Ibrahim Farouk Barakat * and Adel Fathi Hussien**
}

\begin{abstract}
The periapical lesion is the most common sequelae of pulp necrosis due to either carious involvement or trauma. Treatment options to manage periapical lesion with open apex ranges from non-surgical root canal treatment and/or apical surgery to extraction. Objective: clinical and radiographical evaluation of the effect of MTA and bioceramic root canal sealer to manage immature permanent teeth with an open apex. Methodology: The study was composed of sixty teeth from 54 children with ages ranged from seven to ten years old age. Children were divided into two equal groups:_1-Group A: Teeth were received apexification with MTA 2-Group B: Teeth were received apexification with bioceramic root canal sealer. All patients were followed clinically and radiographically immediately postoperatively, 3, and 6 months. Results: The success rate in both bioceramic and MTA was $93.3 \%$ and $90 \%$ respectively with no statistically significant difference. After 6 months, all teeth show normal lamina dura among both bioceramic and MTA groups. While reduced radiolucences and obvious healing of bone detected among $93.3 \%$ and $90 \%$ of teeth among bioceramic and MTA groups respectively. Conclusions: Both MTA and bioceramic sealer show good results
\end{abstract}

KEYWORDS: Apexification, MTA, Bioceramic sealer, nonvital immature permanent teeth.

\section{INTRODUCTION}

Endodontic treatment of immature teeth with incompletely formed roots can result in complications that necessitate special precaution. It is necessary to induce the closure of the apical foramen with mineralized tissue or to create an artificial apical barrier to allow for condensation of the root filling material and promote an apical seal. [1] Apexification is a method to induce a calcified barrier in a root with an open apex or the continued apical development of an incomplete root in teeth with necrotic pulp. In the past, techniques for management of the open apex in non-vital teeth

* Department of Pedodontics and Oral Health, Faculty of Dental Medicine, Al-Azhar University, Cairo, Egypt.

** Preventive Dentistry Department, College of Dentistry, Umm Al-Qura University, Makkah, KSA., Department of Pedodontics and Oral Health, Faculty of Dental Medicine, Al-Azhar University, Cairo, Egypt. 
were confined to custom fitting the filling material, paste fills, and apical surgery. ${ }^{[2]}$ The use of customfitted gutta-percha cones is not advisable as the apical portion of the root is frequently wider than the coronal portion, making proper condensation of the gutta-percha impossible. The sufficient widening of the coronal segment to make its diameter greater than that of the apical portion would significantly weaken the root and increase the risk of fracture. The disadvantages of surgical intervention include the difficulty of obtaining the necessary apical seal in the immature pulpless tooth with its thin, fragile, irregular walls at the root apex. These walls may shatter during the preparation of the retroactivity or condensation of the filling material. The wide foramen results in a large volume of filling material and a compromised seal. Apicoectomy further reduces the root length resulting in a very unfavorable crown root ratio. The limited success enjoyed by these procedures resulted in significant interest in the phenomenon of continued apical development or establishment of an apical barrier, first proposed in the 1960s. ${ }^{[3]}$ Mineral trioxide aggregate (MTA) has been considered the material of choice for endodontic therapies due to its high mineralization capacity and relatively few inflammatory reactions in clinical use. Also, the interactions between MTA and human osteoblast-like cells, cementoblasts, periodontal ligament fibroblasts, and gingival fibroblasts have demonstrated its capability for regenerative endodontic procedures ${ }^{[4]}$ Bioceramics composed of tricalcium silicate, dicalcium silicate, calcium phosphate monobasic, amorphous silicon dioxide, and tantalum pentoxide. Due to their ability to penetrate dentinal tubules and to interact with dentine moisture, an optimum dimensional stability and the least amount of shrinkage can be expected. Compared to white mineral trioxide aggregate (MTA), bioceramics offer the advantage of being aluminum-free and contain tantalum pentoxide as an opacifier. ${ }^{[5]}$ Bioceramics can induce differentiation of human PDL fibroblasts and their bioactivity is comparable with that of MTA. They also have an alkaline $\mathrm{pH}$ (12.8), which can be responsible for antibacterial activity. Bioceramics have many advantages such as biocompatibility, high resistance to wash out, and lack of shrinkage. It also displays good physical properties. The syringe eliminates the need for hand instruments and the need for mixing. The bioceramic particle size is less than $2 \mu$ thus can be delivered by a 0.012 capillary tip which allows premixed material to be placed by syringe. Also, these materials have a compressive strength of 50$70 \mathrm{MPa} \cdot{ }^{[6]}$ However, further studies are necessary to evaluate using these materials for the apical barrier. Therefore, the objective of this study was to evaluate the effect of MTA and bioceramic root canal sealer to manage immature permanent teeth with an open apex.

\section{MATERIALS AND METHODS}

\section{Ethical statement}

The proposal of this in vivo clinical trial was reviewed and ethically approved by the Research Ethical Committee of Faculty of Dental Medicine, Al-Azhar University, Boys' Branch, Cairo.

\section{Sample size}

A sample size of sixty non-vital immature permanent teeth with open apex indicated for apexification from 54 children. 30 teeth in each group have $80 \%$ power to detect an increase of 0.20 with a significance level (alpha) of 0.05 (two-tailed). In $80 \%$ of the power of those experiments, the P-value was less than 0.05 (two-tailed) so the results were deemed statistically significant. In the remaining $20 \%$ of the experiments, the increase was deemed not statistically significant. For sample size calculation, G*Power Software Version 3.1.9.6 Released 2014, (Kiel University, Germany) was used.

\section{Study Participants}

Patients were selected from the out-patient clinic of Pedodontics and oral health department, faculty of Dental Medicine, Al-Azhar University, Boys' 
Branch, Cairo. Teeth were divided into two equal groups as follows: Group A: 30 immature non-vital teeth with open apex were received apexification with MTA. Group B: 30 immature non-vital teeth with open apex were received apexification with bioceramic root canal sealer. Inclusion criteria; The patients were selected after preoperative radiographic evaluation according to the following measures: the child age ranged from seven to eleven years without sex predilection who has nonvital immature permanent teeth with open apex, Cooperative child. Exclusion criteria; Medically compromised patient, uncooperative children. Mobile or non-restorable teeth, vital teeth, or teeth with closed apex.

\section{Apexification procedure}

The procedure was discussed in brief for each patient's parent and informed consent was obtained and signed from them. Before the commencement of the procedure, preoperative intraoral periapical radiographs and findings were recorded.

After administration of the local anesthesia and rubber dam isolation, the cavity outline was established with a sterile \# 330 high-speed carbide bur with air/water spray. The roof of the pulp chamber was removed with a sterile, non-end cutting low-speed bur. The root canals were irrigated with sodium hypochlorite $(\mathrm{NaOCl}) 5 \%$ [7]. The working length of the tooth was determined by the placement of the manual file in the canal, which was estimated from the preoperative radiographs, and confirmed by taking periapical radiographs. Debridement of the canal was performed by K-files. The canals were then irrigated with $\mathrm{NaOCl} 5 \%$ and the teeth were sealed with a temporary filling.

In the second visit, the rubber dam was applied, the canals were irrigated with $\mathrm{NaOCl} 5 \%$ then dried with paper points. According to the obturating materials used, the teeth in Group A were obturated with MTA (Cerkamed medical company, poland). The powder and liquid ratios were $(3: 1)^{[8]}$. Packing of MTA in the canal to fill about $3-5 \mathrm{~mm}$ of the apical part of the canal ${ }^{[9]}$. The teeth in Group B were obturated with a bioceramic root canal sealer (Well-root ST, quality endodontic distributers Ltd, United Kingdom). The syringe tip was adjusted to be shorter than the working length by $1-2 \mathrm{~mm}$ and start to inject bioceramic sealer in the canal and withdrawal the tip of the syringe about $1 \mathrm{~mm}$ and continuous inject until fill about $3-5 \mathrm{~mm}$ of the apical part of the canal. Radiographs were taken to ensure that the material reached the root apex and filled about $3-5 \mathrm{~mm}$. The teeth were temporarily filled for 24 hours to allow the complete set of the sealer. On the next visit, the remaining part of the canals was obturated with gutta-percha (META BIOMED CO., Ltd.,Korea ) till the canals' orifices and the coronal part of the teeth were restored with composite (Compsan LCM, PROMEDICA Neumunster/ Germany).

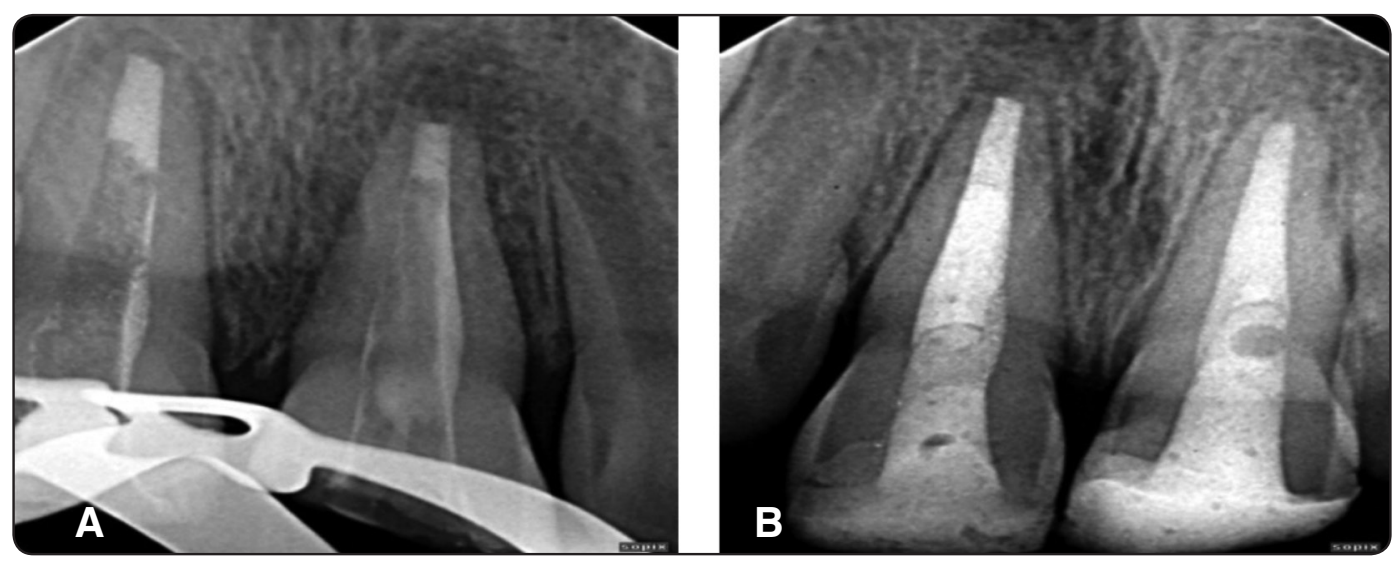

Fig. (A): Apical plug with MTA apical plugin right side and Bioceramic apical plugin left side. Fig. (B): Final restoration with gutta-percha then composite. 
No antibiotics and/or analgesics were administrated during the study period. The pre- and postoperative clinical and radiographical findings were recorded.

\section{Data Management and Statistical Analysis}

The data were collected, tabulated, and statistically analyzed by Statistical Package for social sciences software (SPSS) version 24. A T-test was used for comparing the mean difference between both groups, while the Chi-square test was used for qualitative data. The level of statistical significance was predetermined at a $p$-value of 0.05 .

\section{RESULTS}

A total of 60 immature permanent anterior teeth were treated in this clinical study. The mean age of children included in Group A was $9 \pm 1.20$ years while

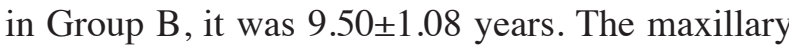
central incisor was the most commonly involved tooth in our study, while only three maxillary lateral incisors were involved in three children. Pulp involvement due to trauma was recorded in $75 \%$ of the affected incisors while $25 \%$ of involvement was due to extensive caries.

The preoperative clinical findings among the study groups are presented in Table (1)

TABLE (1) Distribution of preoperative clinical manifestations among the study groups:

\begin{tabular}{|l|c|c|}
\hline $\begin{array}{l}\text { Preoperative clinical } \\
\text { manifestations }\end{array}$ & $\begin{array}{c}\text { Bioceramic } \\
\mathrm{N}=30\end{array}$ & $\begin{array}{c}\text { MTA } \\
\mathrm{N}=30\end{array}$ \\
\hline Facial ecchymosis and edema & $13.3 \%$ & $10 \%$ \\
\hline Swelling or abscess & $50 \%$ & $63.3 \%$ \\
\hline
\end{tabular}

Regarding the postoperative clinical findings; all participants responded successfully to the intervention and show healing of soft tissue among both bioceramic and MTA groups. Also, swelling or abscess, and ecchymosis and edema were absent among all studied groups (Table 2).

TABLE (2) Postoperative clinical manifestations among the study groups:

\begin{tabular}{|l|c|c|}
\hline Postoperative clinical manifestations & Bioceramic & MTA \\
\hline Facial ecchymosis and edema & $0 \%$ & $0 \%$ \\
\hline Swelling or abscess & $0 \%$ & $0 \%$ \\
\hline Healing of soft tissue & $100 \%$ & $100 \%$ \\
\hline
\end{tabular}

Regarding the preoperative radiological findings, all teeth showed open apex and widening in lamina dura among both bioceramic and MTA groups. While preapical radiolucency was present in $94 \%$ of the teeth among the bioceramic group and $100 \%$ of the MTA group (Table 3).

TABLE (3) preoperative radiological findings among the study groups

\begin{tabular}{|l|c|c|}
\hline Preoperative radiologic findings & Bioceramic & MTA \\
\hline Normal lamina dura & $0 \%$ & $0 \%$ \\
\hline Preapical radiolucency & $94 \%$ & $100 \%$ \\
\hline
\end{tabular}

Regarding the post radiological findings of both groups after 3 and 6 months of follow up, all teeth show normal lamina dura among both groups. While reduced radiolucency and obvious healing of bone detected among $93.3 \%$ and $90 \%$ of teeth among bioceramic and MTA groups respectively Table 4. The distribution of successful and failed cases among the study groups are presented in Fig.1.

TABLE (4) postoperative radiologic findings after 3 and 6 months among the study groups

\begin{tabular}{|l|c|c|c|c|}
\hline \multirow{2}{*}{} & \multicolumn{2}{|c|}{ Bioceramic } & \multicolumn{2}{c|}{ MTA } \\
\cline { 2 - 5 } & After 3 months & After 6 months & After 3 months & After 6 months \\
\hline Normal lamina dura & $100 \%$ & $100 \%$ & $100 \%$ & $100 \%$ \\
\hline Reduced radiolucency & $93.3 \%$ & $93.3 \%$ & $90 \%$ & $90 \%$ \\
\hline
\end{tabular}




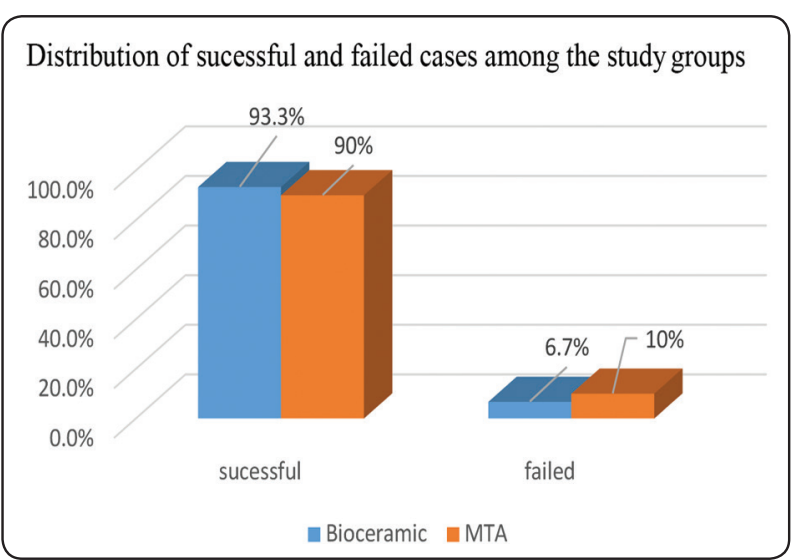

Fig. (1) Distribution of successful and failed cases among the study groups

\section{DISCUSSION}

The periapical lesion is the most common sequelae of pulp necrosis either due to carious involvement or trauma. Many materials have been used successfully for apical closure and periapical repair but the exact mechanism of action is unknown. A permanent treatment is preferable to minimize the chances of reinfection which can result in apical periodontitis and inhibit canal closure. ${ }^{[9]}$

The goal of the current study is to obtain an apical barrier to prevent the passage of toxins and bacteria because dental caries or traumatic injuries may cause microbes to enter the dental pulp. This may cause inflammatory changes in the pulp (pulpitis) that are irreversible. Pulp necrosis (death) is the outcome of irreversible pulpitis, which is a clinical diagnosis based on subjective and objective findings indicating that the clinically normal inflamed pulp is incapable of healing. The susceptibility of microbial invasion of dental pulp due to trauma or dental caries increases after the emergence of the young permanent tooth in the oral cavity, when the roots are still developing. ${ }^{[10-12]}$

In the current study, the apexification technique was used because this technique considers the most reliable model for testing new materials, as it allows them to exhibit their primary effect on the tissue.
Access cavity prepared to allow debridement of the canal, irrigation of the canal with NAOCL 5\% to disinfection and cleaning of the canal. ${ }^{[13]}$

In the present study on regard to the positive response observed in group A, the success rate was approximately (90\%). These results were in agreement with other researchers. Pace R, et al. ${ }^{[14]}$ suggest that apical plug with MTA was a successful and effective technique for long-term management of teeth with necrotic pulps with immature root development and periapical lesions. Johannes $\mathrm{M}$, et al. ${ }^{[15]}$ supported the management of open apical foramina with mineral trioxide aggregate apical plugs. The success rate is due to the good sealing ability of MTA, biocompatibility, and dimensional stability. ${ }^{[16,17]}$ MTA has a $\mathrm{pH}$ of 12.5 after setting which is similar to the $\mathrm{pH}$ of calcium hydroxide and it has been suggested that this may impart some antimicrobial properties ${ }^{[18]}$.

In the present study bioceramic, root canal sealer was used to manage open apex. Bioceramics are highly successful endodontic sealers and have several advantages such as improved biocompatibility, sealing ability, anti-bacterial, ease of application, and an increase in strength of root following obturation. The extreme biocompatibility of bioceramics can also be observed in cases of root repair where there is an absence of inflammation and pain or minimal pain the following overfill during obturation ${ }^{[19]}$. Bioceramics are hydrophilic and can form hydroxyapatite. They lead to the formation of a chemical bond between the filling material and dentin walls. ${ }^{[2]}$ This eliminates the presence of any space between the dentinal walls and sealer which enhances the seal. The bioceramic sealer has a particle size of 2 microns aiding in its delivery by the means of a capillary tip. Bioceramics have shown radiopacity and flow in accordance with ISO $6876 / 2001$ recommendations ${ }^{[21,22]}$.

In the present study on regard to the positive response observed in group $\mathrm{B}$, the success rate 
was approximately $(93,3 \%)$ all teeth showed no signs and symptoms of inflammation clinically. Moreover, 28 from 30 show the absence of periapical and furcation radiolucency, root resorption, and widening of periodontal ligament radio-graphically. This result was in agreement with other researchers. Marga Ree and Richard Schwartz suggest that bioceramic materials are promising material and also a good choice for cases in which extrusion into the periapical tissues may damage vital structures, such as the maxillary sinus or the inferior alveolar nerve. ${ }^{[23]}$ Dasarathan D, Abdullah F, Saravanan P, Davidson D, Manali S, suggest that bioceramic root canal sealer can be considered as a suitable alternative to other materials used in the apexification procedure. ${ }^{[24]}$

In the present study, bioceramic material has several advantages such as better clinical handling properties. The difficulties in the handling of MTA have been frequently reported by clinicians. Another drawback of MTA is the potential for staining dentin, which has been shown in several Vitro studies clinical investigations, and case reports which have shown that both white and gray MTA cause discoloration. To date, there have been no reports of staining of dentin by bioceramic products, which has also been the experience of the authors contain zirconium oxide and tantalum pentoxide as opacifiers. ${ }^{[25-30]}$ The presence of heavy metals may be another potential drawback of MTA. The majority of papers show favorable properties for bioceramic materials including biocompatibility, bioactivity, and antimicrobial properties. It has sealing properties similar to MTA, and some in vitro studies show that bioceramic materials increase resistance to fracture ${ }^{[31,32]}$.

\section{CONCLUSION}

Both MTA and bioceramic root canal sealers recorded successful clinical and radiographical outcomes in the apexification of immature permanent teeth.

\section{REFERENCES}

1. De Jesus Soares A, Yuri Nagata J, Casarin C, Flavio Affonso de Almeida J, Gomes P, Augusto Zaia A, etal. Apexification with new intra-canal medicament: a multidisciplinary case report. Iran Endod J. 2012; 73:165-70.

2. Stewart J. Root canal therapy in incisor teeth with open apices. Br Dent J. 1963;114:249-54.

3. Frank L. Therapy for the divergent pulpless tooth by continued apical formation. J Am Dent Assoc. 1966; 72:87-93.

4. De-Deus G, Canabarro A, Alves G, Linhares A, Senne I, Granjeiro M, et al. Optimal cytocompatibility of a bioceramic nanoparticulate cement in primary human mesenchymal cells. J Endodo. 2009; 35:1387-90.

5. Zhang S, Yang X, Fan M. Bioaggregate and iRoot BP Plus optimize the proliferation and mineralization ability of human dental pulp cells. Int Endod J. 2013; 46: 923-9.

6. Koch K, Brave D, Nasseh A. A review of bioceramic technology in endodontics, CE Article. 2012;4:6-12.

7. Fridlland M, Rosado R. Mineral trioxide aggregate solubility and porosity with different water to powder ratio. J Endod. 2008;29:814-7.

8. Bogen G, Kuttler S. Mineral trioxide aggregate obturation: a review and case series. J Endod. 2009; 35:777-90.

9. Morse R, Lamic O, yesillosy C.Apexification review of literature.1990;21:589-98.

10. Kubasad C, Ghivari B. Apexification with apical plug of MTA-report of cases. Arch Oral Sci Res. 2011;1:104-7.

11. Andreasen O, Paulsen U, Yu Z, Bayer T, Schwartz O. A long-term study of 370 auto transplanted premolars. Part II. Tooth survival and pulp healing subsequent to transplantation. European Journal of Orthodontics 1990; $12: 14-24$

12. Torneck D. Effects and clinical significance of trauma to the developing permanent dentition. Dental Clinics of North America. 1982;26:481-504.

13. Radcliffe E, Potouridou L, Qureshi R, Habahbeh N, Qualtrough A, Worthington $\mathrm{H}$, et al. Antimicrobial activity of varying concentrations of sodium hypochlorite on the endodontic microorganisms Actinomyces israelii, A. naeslundii, Candida albicans and Enterococcus faecalis. Int Endod J. 2004;37:438-46. 
14. Pace R, Giuliani V, Nieri M, Di Nasso L, Pagavino G. Mineral trioxide aggregate as apical plug in teeth with necrotic pulp and immature apices. J Endod. 2014; 40:1250-4.

15. Johannes M ,Nathalie H, Thorsten P, Martin K, Jens D, Hans S, Shimon F. Mineral trioxide aggregate apical plugs in teeth with open apical foramina: a retrospective analysis of treatment outcome. J Endod. 2009;35:1354-8.

16. Torabinejad M, Watson F, Pitt R. Sealing ability of mineral trioxide aggregate when used as a root-end filling material. J Endod 1993;19:591-5.

17. Torabinejad M, Hong U, Lee J. Investigation of mineral trioxide aggregate for root-end filling in dogs. J Endod 1995;21:603-8.

18. Torabinejad M,Hong U, Pitt R, Kettering D. Antibacterial effects of some root-end filling materials. J Endod 1995;21:403-6.

19. Koch K, Brave D. Bioceramic technology - the gamechanger in endodontics. Endodontic Practice US. 2009;12:7-11.

20. Kossev D, Stefanov V. Ceramics-based sealers as new alternative to currently used endodontic sealers. Research Ceramics-based Sealers. 2009;1:42-8.

21. Koch K, Brave D, Nasseh A. A review of bioceramic technology in endodontics, CE Article. 2012;4:6-12.

22. Taccio G, Corriera C, Duarte M, Siqueira D, Gavini G. Evaluation of Radiopacity, $\mathrm{pH}$ release of calcium ions and flow of a bioceramic root canal sealer. Journal of Endodontics. 2012;38:842-5.

23. Marga R and Richard S. Clinical applications of bioceramic materials in endodontics.CE.2014;7:1-9.

24. Dasarathan D, Abdullah F, Saravanan P, Davidson D, Manali S. Management of Non-Vital Teeth with Open
Apex Using Endosequence Root Repair Material, Mineral Trioxide Aggregate and Biodentin - A Case Series. Int J Cur Res Rev. 2017;9:26-30.

25. Belobrov I, Parashos P. Treatment of tooth discoloration after the use of white mineral trioxide aggregate. J Endod. 2011;37(7):1017-20.

26. Boutsioukis $\mathrm{C}$, Noula $\mathrm{G}$, Lambrianidis T. Ex vivo study of the efficiency of two techniques for the removal of mineral trioxide aggregate used as a root canal filling material. J Endod. 2008;34:1239-42.

27. Jang H, Kang M, Ahn S, Kim S, Kim W, Kim Y, Kim E. Tooth discoloration after the use of new pozzolan cement (Endocem) and mineral trioxide aggregate and the effects of internal bleaching. J Endod. 2013;39:1598-602.

28. Maroto M, Barbería E, Vera V, García-Godoy F. Dentin bridge formation after white mineral trioxide aggregate (white MTA) pulpotomies in primary molars. Am J Dent. 2006;19:75-9.

29. Percinoto C, de Castro M, Pinto M. Clinical and radiographic evaluation of pulpotomies employing calcium hydroxide and trioxide mineral aggregate. Gen Dent. 2006;54:258-61.

30. Jacobovitz M, de Lima K. Treatment of inflammatory internal root resorption with mineral trioxide aggregate: a case report. Int Endod J. 2008;41:90512.

31. Zhang H, Shen Y, Ruse D, Haapasalo M. Antibacterial activity of endodontic sealers by modified direct contact test against Enterococcus faecalis. J Endod. 2009; 35: 1051-5.

32. Ulusoy I, Nayır Y, Darendeliler Y. Effect of different root canal sealers on fracture strength of simulated immature roots.Oral Surg Oral Med Oral Pathol Oral Radiol Endod.2011;112: 544-547 